

\title{
Droplet nucleation in miniemulsion thiol-ene step photopolymerization
}

Cuong Minh Quoc Le, Loïc Vidal, Marc Schmutz, Abraham Chemtob

\section{To cite this version:}

Cuong Minh Quoc Le, Loïc Vidal, Marc Schmutz, Abraham Chemtob. Droplet nucleation in miniemulsion thiol-ene step photopolymerization. Polymer Chemistry, 2021, 10.1039/D1PY00139F . hal03173482

\section{HAL Id: hal-03173482 \\ https://hal.science/hal-03173482}

Submitted on 18 Mar 2021

HAL is a multi-disciplinary open access archive for the deposit and dissemination of scientific research documents, whether they are published or not. The documents may come from teaching and research institutions in France or abroad, or from public or private research centers.
L'archive ouverte pluridisciplinaire HAL, est destinée au dépôt et à la diffusion de documents scientifiques de niveau recherche, publiés ou non, émanant des établissements d'enseignement et de recherche français ou étrangers, des laboratoires publics ou privés. 


\title{
Droplet Nucleation in Miniemulsion Thiol-Ene Step Photopolymerization
}

\begin{abstract}
Cuong Minh Quoc Le, ${ }^{\mathrm{a}, \mathrm{b}}$ Loïc Vidal, ${ }^{\mathrm{a}, \mathrm{b}}$ Marc Schmutz, ${ }^{\mathrm{c}}$ and Abraham Chemtob ${ }^{\mathrm{a}, \mathrm{b}}$ *
Complex and unique polymer colloids have been prepared by miniemulsion polymerization through a particle nucleation confined to monomer droplets. Unlike radical chain polymerization, the reaction conditions for a predominant droplet nucleation have not been investigated in step polymerizations such as thiol-ene polymerization. To clarify this issue, an aqueous thiol-ene miniemulsion based on diallyl adipate and 2,2-(ethylenedioxy)diethanedithiol is prepared. Droplet stability is affected by Ostwald ripening due to a limited solubility of hexadecane (costabilizer) in the monomer phase. The control of chemical stability is also difficult, since a self-initiated polymerization is caused by adventitious radicals generated in the emulsification stage (ultrasonication). However, the addition of an appropriate concentration of radical scavenger (quinone) can halt polymerization during several hours. Under these conditions, batch photopolymerization kinetics, molecular weight progress and particle size distribution have been determined with reproducible results. Emphasis is placed on understanding how initiator solubility, droplet size and monomer solubility effect droplet nucleation. For this purpose, a reliable measurement of droplet (particle) size distributions is achieved by combining size data from dynamic light scattering and transmission electron microscopy. When using a water-soluble photoinitiator, a substantial homogeneous nucleation is reported even for highly water-insoluble monomers. Only when droplet diameter is low enough (about $100 \mathrm{~nm}$ ), then droplet nucleation prevails. Conversely, a water-insoluble initiator drives a robust and complete droplet nucleation irrespective of the reaction conditions.
\end{abstract}

Figure 1. Chemical structure of radical photoinitiators, dithiol and diene monomers.

\section{INTRODUCTION}

Radical-mediated thiol-ene chemistry refers to the addition of thiyl radicals to $\mathrm{C}=\mathrm{C}$ double bonds. ${ }^{1}$ Thiol-ene reactions are most commonly photoinitiated, and generally employed for the synthesis of cross-linked poly(thioether) materials via photopolymerization. ${ }^{2}$ The acrylic family of polymers is presently the overwhelming choice in the field of industrial photopolymerization. ${ }^{3,4}$ But, thiol-ene systems have gained acceptance in overcoming the problems associated with monomer toxicity or volume shrinkage. ${ }^{5}$ The result has been the development of niche products, ${ }^{6}$ including for example, non-cytotoxic hydrogels ${ }^{7}$ or low shrinkage dental materials. ${ }^{8}$

Acrylate and methacrylate monomers are also key players in emulsion polymerization, another industrially useful polymerization process. ${ }^{9}$ The resulting acrylic waterborne polymer dispersions are formed by a radical chain polymerization. ${ }^{10}$ The distinguishing characteristic of a thiolene polymerization is that polymer chains grow through a stepwise reaction between ene and thiol functional groups of reactants. ${ }^{11}$ Also, by using a stoichiometric amount of bifunctional ene and thiol reactants, there is evidence that poly(thioether) latexes of practical utility can be formed, i.e. with a molecular weight of about one to several tens of thousands $\mathrm{g} \mathrm{mol}^{-1}{ }^{12}$ However, unlike thiol-ene photopolymers, the share hold by thiol-ene latexes has remained limited, and no commercial product has emerged yet. ${ }^{13,14}$ The main examples result from a miniemulsion photopolymerization, ${ }^{15-}$ 24 and to a less extent, dispersion ${ }^{25,26}$ and emulsion ${ }^{12,27}$ (photo)polymerizations. Suspension photopolymerization has been also described, ${ }^{28-32}$ but this process does not result in polymer colloids (average particle diameter $<1 \mu \mathrm{m}$ ), but in micrometre solid beads $(0.1-10 \mathrm{~mm})$. Although copolymers of acrylic and methacrylic acids and esters account for a major fraction of all industrial latexes, they have significant drawbacks. Among them are the inhibition of the polymerization by oxygen and the chain transfer reactions to polymer that hinders the control of chain structure. ${ }^{33}$ Thiolene polymerization exhibits numerous characteristics that can address these drawbacks, some directly related to its distinct step polymerization mechanism. For example, the thiol-ene reaction is relatively insensitive to oxygen. Further, branching and cross-linking can be accurately tuned by varying the monomer functionality since there is virtually no chain transfer to polymer. ${ }^{34}$ Some authors have already taken advantage of some opportunities, ${ }^{35}$ but further research efforts are required to tap the full potential of thiol-ene step photopolymerization in dispersed media.

The most used process in this field is miniemulsion thiolene polymerization. ${ }^{15-24}$ Involving smaller and therefore less scattering monomer droplets $(50-500 \mathrm{~nm})$ than an emulsion polymerization, it has the added advantage of imparting greater radiation penetration within the reactor. In addition, 


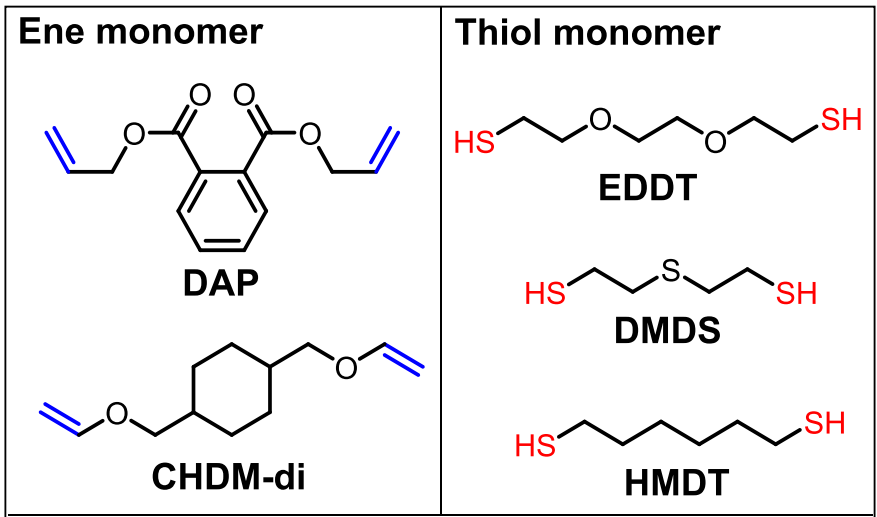

Photoinitiator

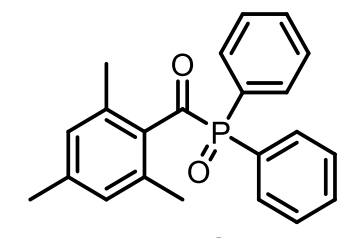

TPO

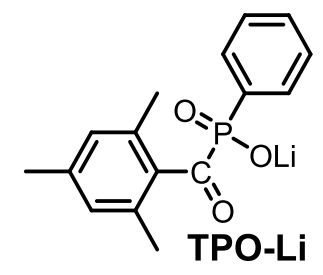

the desired particle nucleation mode occurs via radical capture into the monomer droplets, subsequently converted into polymer particles. ${ }^{36}$ Consequently, when a step polymerization is confined to droplets containing a stoichiometric amount of ene and thiol functional groups, the equilibrium can be driven toward high molecular weights. ${ }^{16}$ However, six years after the first example of thiol-ene miniemulsion photopolymerization, ${ }^{15}$ the question of droplet nucleation efficiency is still largely unanswered. In contrast, there are extensive studies regarding chain radical polymerization in miniemulsion. ${ }^{37-39}$ Regardless of the type of polymerization, the goal of a miniemulsion polymerization is to nucleate most of the initial monomer droplets and minimize homogeneous nucleation; i.e the fact that oligomeric radicals instead of entering into the droplets can precipitate in the aqueous phase to form particles. ${ }^{33}$ The relative extents of droplet and homogeneous nucleation are expected to vary in a thiol-ene step polymerization compared to a conventional radical chain polymerization due to distinct relationships between molecular weight and the percent conversion of monomer. ${ }^{40}$ For fundamental and practical reasons, it is therefore of interest to discuss the set of reaction conditions for the robust droplet nucleation of thiol-ene monomer miniemulsions.

Our study begins with the preparation and the batch photopolymerization of an aqueous model miniemulsion based on diallyl adipate (DAP) and 2,2(ethylenedioxy)diethanedithiol (EDDT) (see chemicals structures in Figure 1) with sodium dodecyl sulfate (SDS) as the surfactant and hexadecane (HD) as the costabilizer. The weight fraction of monomers was kept constant at $20 \mathrm{wt} \%$, and a water-soluble radical photoinitiator (TPO-Li) of the monoacylphosphine oxide family was chosen. Using an appropriate concentration of radical scavenger (quinone), it was possible to achieve a chemically stable EDDT-DAP miniemulsion. Under these conditions, droplet stability, polymerization kinetics, molecular weight progress and particle size distribution could be determined. However, it was difficult to adequately discuss the mechanism of particle nucleation with this system because of the limited droplet stability. Upon decreasing the weight fraction of monomer to 1 wt\%, monomer droplets possessing photolatency, size monodispersity and stability were eventually obtained. Emphasis was placed on understanding the reaction parameters which are important in favouring droplet nucleation, i.e., droplet size, initiator solubility and monomer solubility. ${ }^{33}$ Initiators and thiol-ene monomers of different chemical structures have been used for this purpose (see Figure 1 for details). Comparison of particle size (number) and droplet size (number) was exploited to evaluate qualitatively the extent of droplet and homogeneous nucleation. ${ }^{41}$ However, a major problem is generally the lack of precision and accuracy in the determination of droplet and particle size distribution, possibly leading to erroneous conclusions about the nucleation mode. ${ }^{42}$ To minimize interpretations errors, the size distribution determined by dynamic light scattering (DLS) was compared with the one given by transmission electron microscopy (TEM).

\section{EXPERIMENTAL SECTION}

\section{Materials}

Diallyl phthalate (DAP, > 98\%, water solubility: 0.15 $\mathrm{mg} / \mathrm{mL}$ ), 2,2-(ethylenedioxy)diethanedithiol (EDDT, > 97\%, water solubility: $11.4 \mathrm{mg} / \mathrm{mL}$ ), 1,6-hexanedithiol (HMDT, > $97 \%$, water solubility: $0.15-0.27 \mathrm{mg} / \mathrm{mL}), \quad 2,5$-di-tertbutylhydroquinone (DBHQ, > 98\%), pyrogallol (PY, 99\%), sodium dodecyl sulfate (SDS, 98\%), hexadecane (HD, > 98\%) lithium phenyl(2,4,6-trimethylbenzoyl)phosphinate (TPO-Li, > 99\%) and diphenyl(2,4,6-trimethylbenzoyl)phosphine oxide (TPO, > 98\%) were purchased from TCl Chemicals. 2,2'thiodiethanethiol (DMDS, > 95\%, water solubility: $0.62 \mathrm{mg} / \mathrm{mL}$ ) is a gift from Bruno Bock. 1,4-bis[(vinyloxy)methyl]cyclohexane (CHDM, > 98\%, water solubility $0.025 \mathrm{mg} / \mathrm{mL}$ ) is a gift from BASF. Dimethyl sulfoxide- $d^{6}$ (DMSO- $d^{6}, 99.5 \%$ D) was bought from Eurisotop. All chemicals were used without further purification.

\section{Synthesis}

Miniemulsion thiol-ene photopolymerization was prepared as follow. A monomer phase containing EDDT (1.70 g, 9.2 $\mathrm{mmol}$ ), DAP (2.3 g, $9.2 \mathrm{mmol}), \mathrm{DBHQ}(40.5 \mathrm{mg}, 50 \mathrm{mM}$ with respect to monomer), and $\mathrm{HD}(0.16 \mathrm{~g})$ was mixed in a $25 \mathrm{~mL}$ vial. The organic phase was then mixed with $16 \mathrm{~mL}$ of an aqueous phase containing SDS $(0.14 \mathrm{~g}, 3.5 \mathrm{wt} \% / \mathrm{monomer}, 30$ $\mathrm{mM}$ in water). A macroemulsion was formed by high-speed mixing with Ultra-Turrax setting at $20000 \mathrm{rpm}$ for $5 \mathrm{~min}$. The resulting macroemulsion was further emulsified by using a Branson sonifier SFX250 for $5 \mathrm{~min}$ at $90 \%$ amplitude and pulse mode $5 \mathrm{~s}$ ON / $1 \mathrm{~s}$ OFF cycles. A portion of $10 \mathrm{~g}$ of miniemulsion was mixed with $2 \mathrm{~mL}$ of a TPO-Li solution (20 $\mathrm{mg} / \mathrm{mL}, 2 \% \mathrm{w} / \mathrm{w}$ respect to monomer) in a $20 \mathrm{~mL}$ cylinder vial. 
The sample was placed at the center of a circular photoreactor fabricated by wrapping a 3 meter of UV-LED strip (SMD3528 type, 60 LEDs $/ \mathrm{m}, 385-390 \mathrm{~nm}$ ) onto inner-wall of a quartz cylinder (ID $80 \mathrm{~mm} \times \mathrm{H} 100 \mathrm{~mm}$ ). The sample was irradiated for $20 \mathrm{~min}$ at room temperature while maintain stirring at 1100 $\mathrm{rpm}$ (irradiance $=3.7 \mathrm{~mW} / \mathrm{cm}^{2}$ ).

\section{Characterization}

${ }^{1} \mathbf{H}$ NMR. The chemical stability of monomeric miniemulsion after sonication was monitored by ${ }^{1} \mathrm{H}-\mathrm{NMR}$ spectroscopy. At a determined time, a $30 \mu \mathrm{L}$ aliquot was diluted in $570 \mu \mathrm{L}$ DMSO- $\mathrm{d}^{6}$ for measurement of ene conversion. Integral areas for the methylene protons at 4.7 ppm and $4.25 \mathrm{ppm}$, corresponding to the proton in monomer and polymer product, were used to evaluate ene-conversion.

Turbiscan. The droplet stability of the EDDT-DAP miniemulsions was measured at $30{ }^{\circ} \mathrm{C}$ in a Turbiscan LAbexpert (Formulaction). The thiol-ene miniemulsion was transferred to a customized glass vial designed for Turbiscan analyser and loaded into the instrument. A near-infrared light source (880 $\mathrm{nm}$ ) was sent to a sample and the backscattered signal (BS) $\left(45^{\circ}\right.$ from the incident radiation) was recorded as a function of position (from top to bottom of the vial), enabling the detection of the migration phenomena. Then BS signal was collected as a function of storage time. The measured BS signal subtracted with the first measurement (time zero) yielded $\triangle \mathrm{BS}$ (\%). All BS signals were calibrated with Teflon standards. The backscattered light, which depends on the droplet mean diameter and on the monomer weight fraction, provides a useful measurement of the droplet stability.

DLS measurement. Droplet and particle sizes were measured by DLS using a VASCO particle size analyser (Cordouan technologies), operating at $658 \mathrm{~nm}$ laser. The intensity of the scattered light was detected at an angle of $135^{\circ}$. Unless otherwise mentioned, the sample was diluted in water to obtain $0.1 \%$ solid content before each measurement. NanoQ software version 2.6 was employed for processing DLS data, the cumulant method was applied to determine the intensity-weighted mean diameter (z-average) and polydispersity index $P d l^{D L S}$. All the samples were measured at least six times and the average value was presented with its standard deviation. For size distribution measurements of monomer miniemulsions (monomer weight fraction: 1 and 20 wt\%), $20 \mu \mathrm{L}$ samples were placed on the measurement cell and the Dual Thickness Controller (DTC) was set as DOWN mode, resulting in a thin film of miniemulsion of approximate $100 \mu \mathrm{m}$ thickness (factory setting).

Transmission electron microscopy (TEM). In cryo-TEM analysis, a drop of $5 \mu \mathrm{L}$ sample $(0.1-1 \mathrm{wt} \%)$ was deposited on copper grid covered with carbon film which was rendered hydrophilic using an ELMO glow discharge unit (Cordouan Technologies, France). The grid was placed in a home-made freezing chamber maintained at $22^{\circ} \mathrm{C}$ and a relative humidity at $80 \%$ before plunging into liquid ethane held at $-190{ }^{\circ} \mathrm{C}$ by liquid nitrogen. The grid was mounted onto a cryo holder (Gatan 626, Pleasanton, CA, USA) and observed under low dose conditions in a Tecnai G2 microscope (FEl, Eindhoven, Netherland) at $200 \mathrm{kV}$. Images were acquired using an Eagle slow scan CCD camera (FEI). In negative stain TEM analysis (NSTEM), a drop of $5 \mu \mathrm{L}$ ethanol was applied onto copper grid covered with a plain carbon film for $1 \mathrm{~min}$. The excess solvent was drained by touching the grid to the edge of a filter paper. Then a drop of $5 \mu \mathrm{L}$ latex sample (0.1 wt\%) was applied. After 1 min of interaction, the excess was removed, and the residue was immediately stained by $5 \mu \mathrm{L}$ staining solution ( $2 \mathrm{wt} \%$ sodium phosphotungstate in water) for $1 \mathrm{~min}$. The grid was fully dried by filter paper. The NS-TEM images were obtained by a JEOL ARM-200F (JEOL, Tokyo, Japan) instrument working at $200 \mathrm{kV}$. The images were recorded with a Gatan camera (Orius 1000 model). Regardless of the technique, the number of particles were counted by using ImageJ software. The number-average diameters $\left(D_{n}=\sum n_{i} D_{i} / \sum n_{i}\right.$, where $n_{i}$ is the number of particles of diameter $D_{i}$ ) and volume-average diameters $\left(D_{v}=\sum n_{i} D_{i}^{4} / \sum n_{i} D_{i}^{3}\right)$ were calculated. Polydispersity index $P d I^{T E M}=\left(\sigma / D_{n}\right)^{2}$ is defined as the squared ratio of the standard deviation $(\sigma)$ of the particle diameter distribution divided by the number-average diameter. $P d l^{T E M}$ was used to estimate the width of size distribution.

Size exclusion chromatography (SEC). Molecular weight and its polydispersity were measured by SEC (Agilent 1260 Infinity) using THF as an eluent. The solvent was pumped through a set of columns (Polymer Laboratories ResiPore, nominal particle size: $3 \mu \mathrm{m}$; porosity: $2 \mu \mathrm{m})$ composed of a guard column $(50 \times 7.5 \mathrm{~mm})$ and two columns $(300 \times 7.5 \mathrm{~mm})$. The flowrate was set at $1 \mathrm{~mL} / \mathrm{min}$ and the column temperature was controlled at $35^{\circ} \mathrm{C}$. The system was then calibrated using universal calibration standards (EasiVial polystyrene standards from Agilent). The dried latexes were dissolved in THF to give a final concentration of $5 \mathrm{mg} / \mathrm{mL}$ and filtered through a $0.2 \mu \mathrm{m}$ PTFE membrane before injection. The Agilent GPC/SEC software and multi-detector were used to determine the molecular weight data.

\section{RESULTS AND DISCUSSION}

\section{Miniemulsion Step Photopolymerization of EDDT-DAP}

I.1 Chemical stability of monomer miniemulsion 


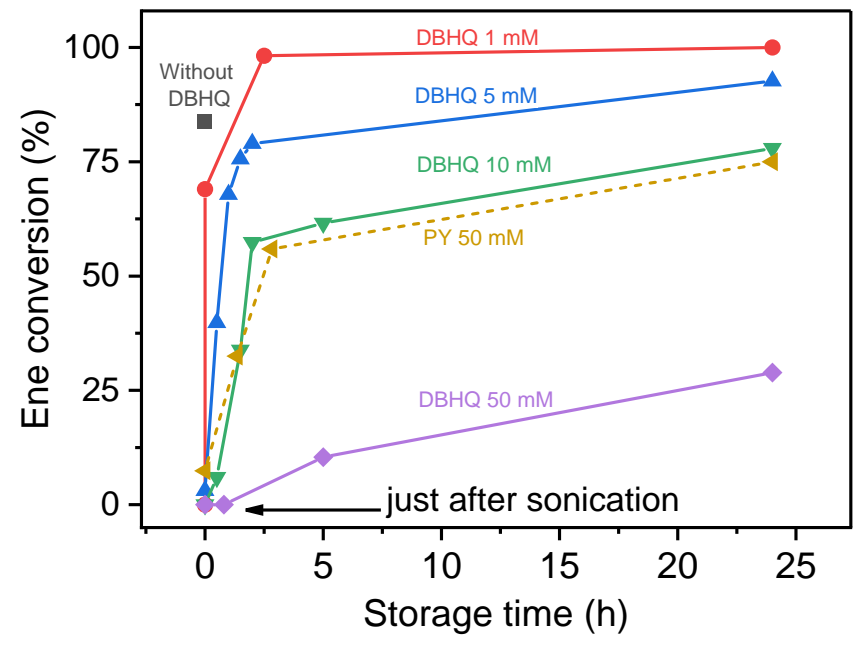

Figure 2. Ene conversion as a function of storage time of an EDDT-DAP aqueous miniemulsion ( $20 \mathrm{wt} \%$ monomer phase content), $\mathrm{t}=0$ is the end of ultrasonication.

Miniemulsions containing a weight fraction of $20 \mathrm{wt} \%$ in DAP (diene) and EDDT (dithiol) monomers have been obtained in three stages: firstly, the preparation of an organic monomer phase including HD as a costabilizer (against Ostwald ripening), secondly, its coarse dispersion in an aqueous phase surfactant continuous phase, and thirdly, its emulsification using ultrasonication to form fine droplets typically in the 50- 500 $\mathrm{nm}$ size range. There are many reports in the literature about the limited shelf-life stability of thiol-ene systems, generally in bulk without the use of solvent. ${ }^{43-46}$ The reasons are their very rapid polymerization rates and sensitivity to the presence of small concentrations of impurities and other adventitious radicals. The situation is even more complex for a thiol-ene miniemulsion because it involves a multicomponent system and several preparation steps. In spite of these problems, a chemical stability is desired for the polymerization to be temporarily controlled. In addition, a premature reaction may affect droplet break-up and droplet size distribution by changing the composition and viscosity of the dispersed phase. $^{47}$

Figure 2 shows the temporal evolution of ene conversion (see ${ }^{1} \mathrm{H}$ NMR data in Fig. S1 of Supporting Information SI) in the dark of a EDDT-DAP miniemulsion, taking the end of ultrasonication as the initial time of measurements. Using an initiator-free system, the functional group consumption stands at $84 \%$ just after sonication with a standard deviation of $15 \%$ (the experiment was replicated 3 times). It is extremely difficult to obtain reproducible kinetic data because polymerization proceeds at very rapid rates. Control measurements of monomer conversion after each preparation stage shows that the main source of premature reaction is the last step of ultrasonication. In the first steps, EDDT-DAP mixture in bulk or dispersed in water can be also subjected to spontaneous reaction, but at much lower conversion rates than the sonicated samples. The ability of ultrasonication to cause radical polymerizations has been reported in the literature. ${ }^{48}$ Ultrasonic cavitation generates very high local temperatures and pressures, resulting in bond breakage and the formation of radicals, such as hydroxyl radicals $\left(\mathrm{OH}^{\circ}\right)$ when it proceeds in water. ${ }^{49}$ Although difficult to solve, the problem of spontaneous thiol-ene reaction may be circumvented by the addition of phenolic radical inhibitors added to the organic phase.

Two types of radical stabilizers of different solubility in aqueous and organic phase - pyrogallol (PY) and 2,5-di-tertbutylhydroquinone (DBHQ) - were evaluated by comparing their ability to prevent miniemulsion polymerization. ${ }^{50} \mathrm{PY}$ and DBHQ have partition coefficients $(\log P)$ of 0.79 and 2.44 respectively, suggesting different locations. While DBHQ is mostly encapsulated in monomer droplets, PY is distributed between the two phases. As shown in Figure 2, when used at similar concentration (50 $\mathrm{mM}$ with respect to monomer), the PY-stabilized miniemulsion exhibits a conversion of $30 \%$ after 1 $\mathrm{h}$ storage time while DBHQ-stabilized analogue is almost unconverted. Note that 4-hydroxyphenol is another effective radical stabilizer for thiol-ene miniemulsion employed by Patton et al. ${ }^{17}$ Consequently, DBHQ was used as radical inhibitor in the rest of the study. The other challenge is to use it at minimal concentration to limit detrimental effects on the final values of monomer conversion and molecular weight. Figure 2 depicts the ene conversion as a function of storage time for a range of DBHQ concentrations ( $1-50 \mathrm{mM})$. There is a trend indicating a better shelf-stability upon increasing the inhibitor content. At $50 \mathrm{mM}$ of $\mathrm{DBHQ}$, monomer conversion remains at $0 \%$ just after sonication, and is less than $10 \%$ within the next five hours. For a monomer miniemulsion, preserving the chemical stability in the order of several minutes to several hours is generally sufficient to give time for the operator to conduct polymerization and to obtain reproducible results.

\section{I.2 Monomer droplet stability}

Stability of a miniemulsion droplet is achieved provided that the destabilization rate by coalescence and Ostwald ripening are sufficiently low. ${ }^{36}$ Kinetically stable monomer droplets (i.e., having both colloidal and diffusional stability) can be obtained by appropriate choice/concentration of surfactant and costabilizer. Few detailed stability studies of thiol-ene monomer miniemulsions have been reported in the literature. ${ }^{16}$ A stability study based on Turbiscan data measuring backscattered light over storage time is presented in Figure 3A for a EDDT-DAP miniemulsion with or without radical inhibitor. In the DBHQ-based monomeric miniemulsion, a change in backscattered signal ( $\triangle \mathrm{BS}$ ) occurred in the matter of minutes, indicating a poor stability. The constantly fluctuating backscattering signal arises because the limited chemical stability may interact in a complex manner with droplet stability. This system is, therefore, not a true miniemulsion in the sense that it is not stable over a period of weeks or months. However, the destabilization is slow and moderate (without apparent phase separation), which implies that a photopolymerization remains possible if it is performed just after sonication. Conversely, the stability of the miniemulsion was strongly improved in the absence of radical inhibitor, and this latter was stable for at least $5 \mathrm{~h}$. This miniemulsion contains a significant fraction of polymer because of the self-initiated polymerization induced by 

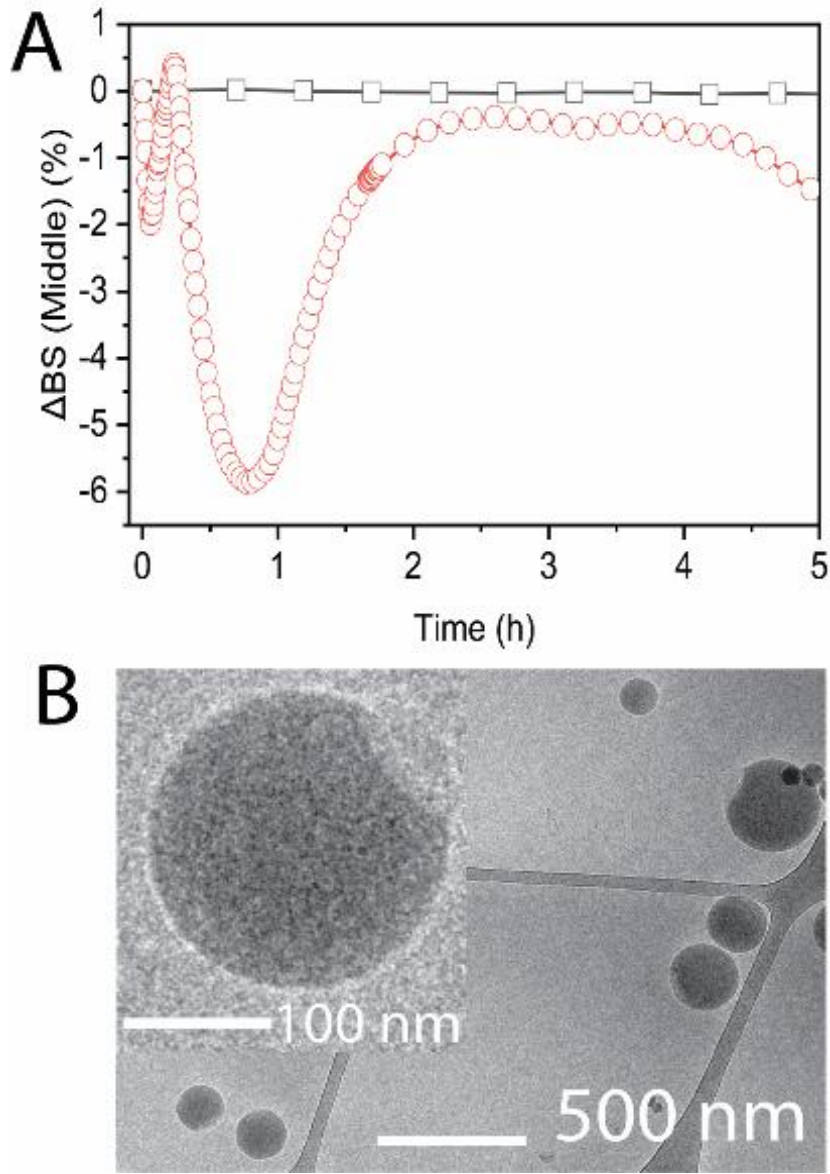

Figure 3. (A) Variation of backscattered signal $(\triangle B S)$ in the middle of the measuring vial as a function of storage time for an EDDT-DAP miniemulsion prepared without DBHQ (square) and with $50 \mathrm{mM}$ DBHQ as radical stabilizer (circle). (B) cryo-TEM image of an EDDT-DAP miniemulsion containing DBHQ, implying that the observed monomer droplets do not contain polymer.

sonication discussed previously. It is known that the inclusion of a water-insoluble monomer-soluble polymer can significantly reduce diffusional degradation, ${ }^{51}$ and therefore, explains that a kinetically stable miniemulsion could be produced in this case. The fact that the droplet stability depends on the in situ formed polymeric costabilizer points out that Ostwald ripening is the predominant destabilization process in the first unreacted miniemulsion.

Surprisingly, changing HD concentration did not improve the stability of the DBHQ-based miniemulsion (see Fig. S2), although $H D$ is known to be a very effective costabilizer in the literature. ${ }^{52}$ The limited solubility of HD in EDDT-DAP monomer mixture may account for the poor costabilizing properties. Not easily evidenced at the macroscopic level, the insolubility could be revealed through a cryo-TEM image of the monomer droplets. Figure 3B shows a typical image of an EDDT-DAP miniemulsion. The droplets have a broad size distribution (60$350 \mathrm{~nm}$ ) and a number-average diameter of approximately 152 $\mathrm{nm}$. At higher magnification, one can observe in most droplets the existence of a separate and brighter HD phase. Similar phase segregation into a Janus structure has been observed previously in immiscible polymer blends and microspheres. ${ }^{53}$ In comparison to a core-shell architecture, an anisotropic biphasic morphology is more thermodynamically favourable due to minimization of interfacial surface area. ${ }^{54}$ In addition to HD phase separation, the water-solubility of EDDT $(15 \mathrm{mg} / \mathrm{mL}$ at $25^{\circ} \mathrm{C}$ ) is thought to be another contributing factor to the Ostwald ripening.

\section{I.3 Polymerization kinetics and molecular weight progress}

A photopolymerization was performed with a $50 \mathrm{mM}$

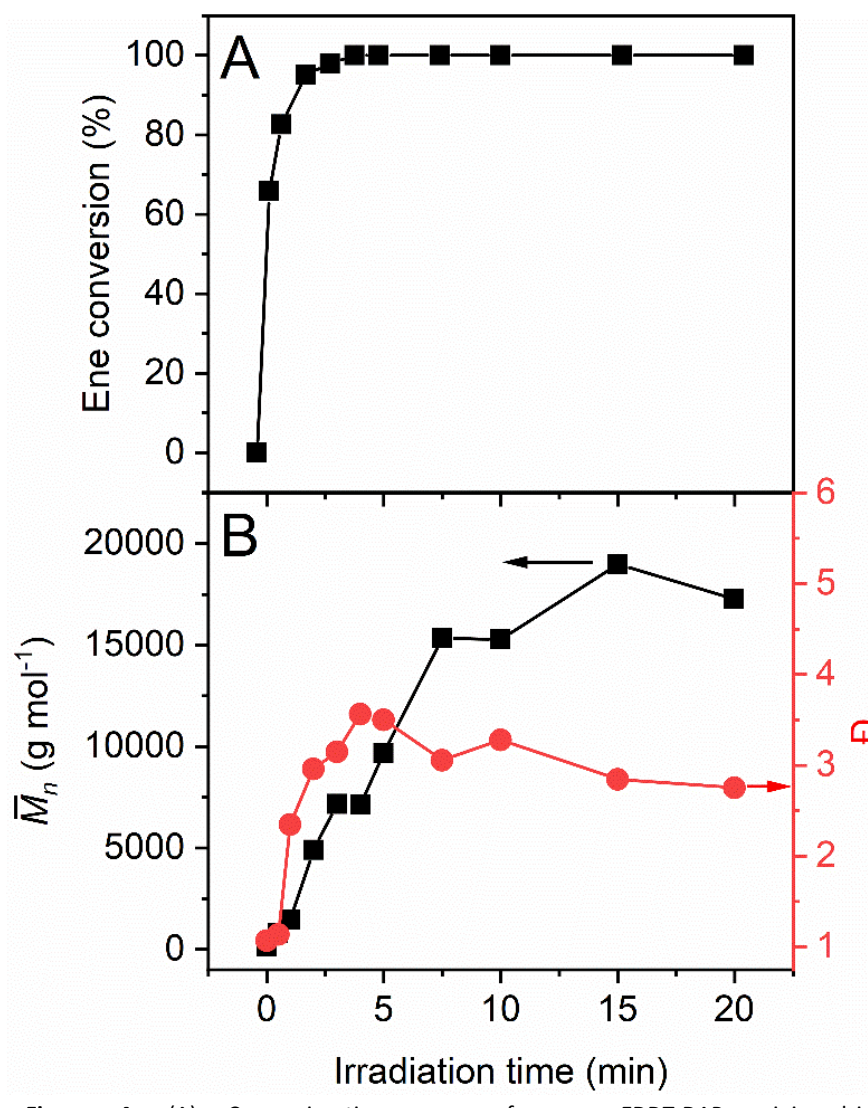

Figure 4. (A) Conversion-time curve for an EDDT-DAP miniemulsion photopolymerization using the water-soluble photoinitiator TPO-Li. (B) Dependence of number-average molecular weight $\left(\overline{\boldsymbol{M}}_{\boldsymbol{n}}\right)$ and dispersity $(\Theta)$ on irradiation time. Monochromatic LED irradiation at $385 \mathrm{~nm}, \mathrm{I}=3.7 \mathrm{~mW} / \mathrm{cm}^{2}$.

DBHQ-stabilized EDDT-DAP miniemulsion (just after ultrasonication) containing the water-soluble photoinitiator TPO-Li. The conversion-time curve is shown in Figure 4A, it was obtained from a series of ${ }^{1} \mathrm{H}$ NMR spectra at different irradiation times (see Fig. S3 of SI). Despite high scattering of miniemulsion droplets and low irradiance of LED light (3.7 $\mathrm{mW} / \mathrm{cm}^{2}$ ), a full conversion was achieved in less than $5 \mathrm{~min}$. Note that the reaction was extended to $20 \mathrm{~min}$, however, to favour the formation of high-molecular-weight polymer that in step-growth polymerization is only significant at the last percentage of reaction conversion. Despite the high concentration of radical inhibitor, the polymerization kinetic remains fast without any apparent induction period. The reasons lie on the initiation efficiency and the high addition $\left(k_{P}\right)$ and hydrogen transfer $\left(k_{T}\right)$ rate constants governing the thiol-ene polymerization of DAP and EDDT monomers. The values of $k_{P}$ and $k_{T}$ are in the range of $10^{4}-10^{6} \mathrm{~L} \mathrm{~mol}^{-1} \mathrm{~s}^{-1}$ at 


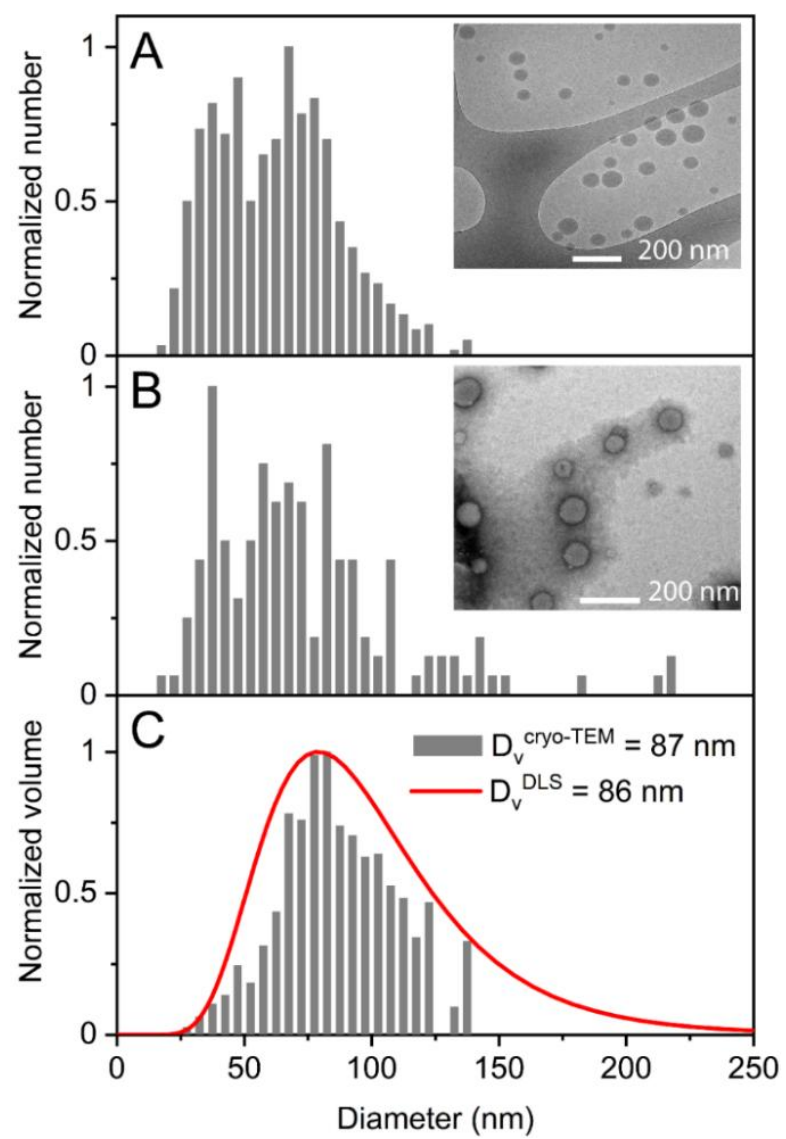

Figure 5. (A) Cryo-TEM image of a latex obtained via EDDT-DAP miniemulsion photopolymerization and the corresponding number-weighted size distribution. (B) NS-TEM image of the same latex and the derived particle number size distribution. (C) Comparison of volume-weighted size distributions derived from DLS and cryo-TEM for a latex based on EDDT-DAP. The standard deviations for the volume-weighted diameters are $2.0 \mathrm{~nm}\left(D_{v}{ }^{\text {CYo-TEM }}\right)$ and $2.6 \mathrm{~nm}\left(D_{v}{ }^{\text {DLS }}\right)$.

ambient temperature, typically in the same range as the propagation rate constants of conventional acrylates. ${ }^{55}$

To investigate the evolution of molecular weight during photopolymerization, aliquots of the reaction medium were withdrawn at defined intervals between $30 \mathrm{~s}$ and $20 \mathrm{~min}$, quenched with DBHQ before SEC analysis. Figure 4B shows the growth of number-average molecular weight $\left(\bar{M}_{n}\right)$ of polysulfide samples and molecular weight dispersity $(\theta)$ as a function of irradiation time (the series of SEC traces is given in Fig. S4 of SI). The polymerization reaction has a typical kinetic profile of a conventional step-growth polymerization, in which high molecular weight is only obtained at very high conversion. At the end of the reaction, a polymer with $\bar{M}_{n}=17,000 \mathrm{~g} \mathrm{~mol}^{-1}$ and $\emptyset=2.8$ was achieved, which are reasonable values for a step-reaction polymerization.

\section{I.4 Colloidal properties of polymer particles}

Due to their sub-ambient glass transition temperature, poly(thioether) particles are not adapted to analysis by conventional TEM. This technique involves the drying of the latex, with possible alterations of particle shape and size. ${ }^{56}$ By contrast, negative staining (NS) and cryo-TEM have been found to be more appropriate than conventional TEM for the observation of soft particles. ${ }^{57}$ As shown in the cryo-TEM pictures of Figure $\mathbf{5 A}$, the particles derived for EDDT-DAP polymerization were homogeneous with a spherical shape. A significant number of particles (>700) were counted for estimating a number-weighted mean diameter $\left(D_{n}^{\text {cryo-TEM }}\right)$. As shown in the size distribution plot, $D_{n}^{\text {cryo-TEM }}$ was evaluated at $63 \mathrm{~nm}$ with a relatively broad size distribution $\left(P D I^{\text {cryo-TEM }}=0.14\right)$. Figure $5 \mathrm{~B}$ is an image of the same latex sample, but negatively stained with phosphotungstic acid. As indication of successful negative staining, the particles appear as low contrast objects with a darker halo surrounding them. ${ }^{58}$ Consistently, a negative stain does not penetrate the object, but coats the surface, obscuring the object itself and all internal structural details, and resulting in such characteristic footprint like aspect. As can be seen in Figure 5B, the numberweighted mean diameter $D_{n}^{\text {NS-TEM }}(73 \mathrm{~nm})$ calculated from more than 150 particles is relatively consistent with those given by cryo-TEM. The slight deviation has been ascribed to the presence of few larger particles (> $150 \mathrm{~nm}$ ) resulting in a broader size distribution $\left(P D I^{N S-T E M}=0.25\right)$. Their absence in the cryo-TEM picture means that the negative stained object may suffer locally from a dehydration followed by coalescence.

An intensity-weighted mean diameter, also referred to as z-average diameter, was typically obtained by DLS (using the conventional cumulant method). Using the same poly(thioether) sample, Figure $\mathbf{5 C}$ shows an intensity-weighted mean diameter $\left(D_{i}^{\text {DLS }}\right)$ of $93 \mathrm{~nm}$ and a broad size distribution $\left(P D I^{D L S}=0.18\right)$. We should note that this diameter cannot be compared directly with a number-weighted mean diameter derived from electron microscopy measurements (although they are both hydrodynamic diameters). This difference and the broad size distribution of the sample can explain the larger particle size found in DLS compared to TEM. To make a comparison possible between the two methods, the DLS intensity-weighted diameters and the TEM number-weighted diameters were both converted to volume-weighted mean diameters $\left(D_{v}\right)$, the former using the Mie theory, and the latter by calculation (see experimental section for details). As shown in Figure 5C, a good agreement was found between the two volume-average size distributions given by cryo-TEM and DLS analysis.

\section{I.5 Approaching particle nucleation mode}

Investigating droplet nucleation requires the availability of reliable droplet and particle size data. ${ }^{33}$ In the case of EDDTDAP system, the latter can be obtained but not the former because of the limited colloidal stability of the monomeric miniemulsion discussed previously. Under these conditions, the reader is cautioned that the droplet size data are not reliable enough. Misinterpretation of DLS size data due to poor monomer miniemulsion stability can have a significant impact on the conclusions that are reached with regard to the nucleation mode. Nevertheless, a first reasonable approach for investigating particle formation mechanism involves comparing only the DLS size distributions of polymer particles and monomer droplets. As depicted in Figure 6A, the two plots show Gaussian distributions appreciably shifted, with limited 
overlap. This suggests that the general trend is a predominant homogeneous nucleation over droplet nucleation.

In addition, the reaction was accompanied by a sharp drop of the backscattered signal $\triangle B S$ as can be seen in Figure $6 \mathbf{B}$. Such evolution of optical properties can be reconciled with the course of a polymerization where homogeneous nucleation plays a major role. In such case, the nucleated particles have a limited concentration in monomer, and their growth proceeds by mass transfer from the non-nucleated droplets acting as reservoir like in the same manner as for emulsion polymerization. Light scattering intensity drops as a result of the disappearance of monomer droplets, which are significantly larger than the particles. In the case of a predominant droplet nucleation, the expected similarity between droplet and particle size distributions would imply rather that the scattering properties are essentially unchanged.
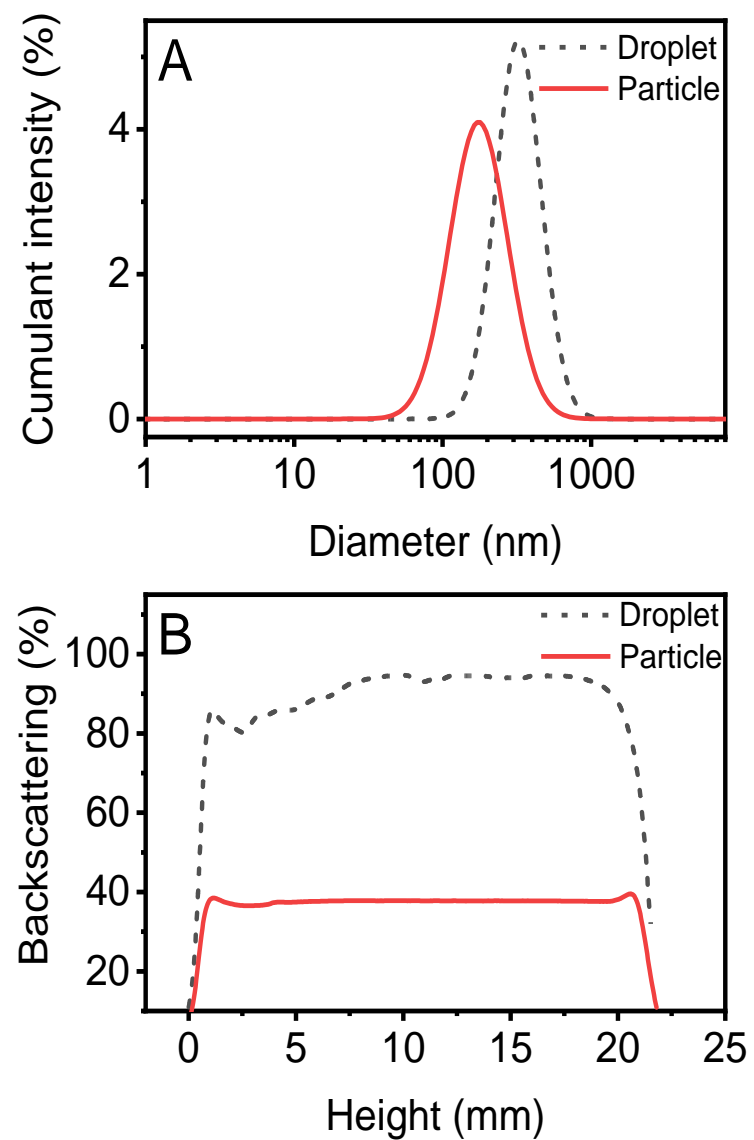

Figure 6. (A) DLS size distributions in the EDDT-DAP miniemulsion photopolymerization. Dotted line: monomer (before irradiation); solid lines: particles (after polymerization, $20 \mathrm{~min}$ at ambient temperature). (B) Evolution of backscattering signal as measured by Turbiscan.

\section{Investigation of Droplet Nucleation}

\section{II.1 Methodology}

To study accurately how the particles are formed, it is necessary to start the miniemulsion polymerization with photolatent, stable and monomodal thiol-ene monomer droplets. In our case, a decrease of the monomer weight fraction was the only way of meeting these three requirements. Stable EDDT-DAP droplets could be generated by means of a monomer weight fraction of $1 \mathrm{wt} \%$. Similarly, the miniemulsion was emulsified using SDS, costabilized by HD, and made chemically inert by DBHQ. It is useful to mention that SDS concentration was kept at $6.0 \mathrm{mM}$, below CMC, so that the contribution of micellar nucleation is neglected. Evidence for the formation of a kinetically stable monomeric miniemulsion is based on DLS and Turbiscan data provided in SI (Fig. S5 in SI). In these conditions of droplet stability, one can safely examine the effect of three experimental parameters known to have a significant impact on droplet nucleation: initiator solubility, initial droplet size and monomer solubility. By comparing droplets and particles with regard to their size distribution and number (the concentration of polymer particles in units of number of particles per litre) in each case, it is possible to ascertain whether droplet nucleation occurs, and, if it does, its extent relative to homogeneous nucleation.
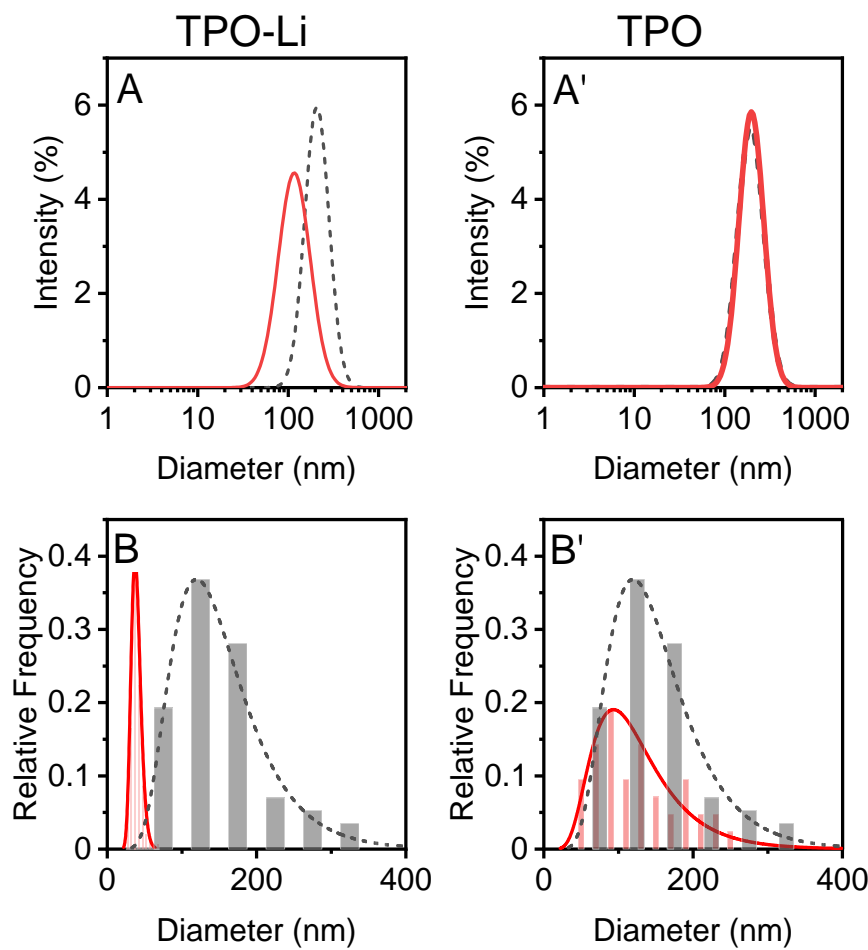

Figure 7. DLS (top) and TEM (bottom) size distributions in a EDDT-DAP miniemulsion photopolymerization with a weight fraction in monomer of $1 \mathrm{wt} \%$ using TPO-Li (A, B) or TPO $\left(A^{\prime}, B^{\prime}\right)$ as initiator. Dotted line: monomer droplets; solid line: polymer particles. In TEM data ( $B$ and $\left.B^{\prime}\right)$, the droplet size distributions were determined using cryo-TEM while particle size distribution derived from NS-TEM.

\section{II.2 Effect of initiator solubility}

Two radical photoinitiators from the same monoacylphosphine oxide family were employed: diphenyl(2,4,6-trimethylbenzoyl)phosphine TPO as oil-soluble photoinitiator and the water-soluble salt analogue TPO-Li used previously (see chemical structures in Figure 1). Figure 7 compares the size distributions of droplets and particles in each case and using two independent methods: DLS (top) and TEM (bottom). An advantage of using miniemulsions with low monomer concentration in DLS is the absence of the need for 



Figure 8. Droplet size distribution (dotted line) and particle size distribution (solid line) in EDDT-DAP miniemulsion photopolymerization (monomer content: $1 \mathrm{wt} \%$ ) using TPOLi (left column), and TPO (right column); at $[S D S]=3 \mathrm{mM}\left(A, A^{\prime}\right), 6 \mathrm{mM}\left(B, B^{\prime}\right), 12 \mathrm{mM}$ $\left(C, C^{\prime}\right)$, and $24 \mathrm{mM}\left(\mathrm{D}, \mathrm{D}^{\prime}\right)$.

dilution, thus limiting the risk of monomer dissolution and droplet size variation. Regarding TEM, particle size data were collected from NS TEM images (Fig. $\mathbf{S 6}$ in SI), while droplet size data were obtained by cryo-TEM analysis. Regardless of the initiator solubility, the starting miniemulsion is monomodal and has an intensity-average diameter of about $175 \pm 11 \mathrm{~nm}$.
The nucleation is completely different whether the initiator is water-soluble or not. When using TPO-Li (A for DLS data and B for TEM data), the size distribution of the droplets significantly deviates from that of the particles irrespective of the method used. The ratio of number of particles over number of droplets $\left(N_{p} / N_{d}\right)$ was estimated at 8.2 (DLS data). Homogeneous nucleation appears therefore to be the predominant particle nucleation process, but droplet nucleation may be very slightly present. The good agreement of DLS (A) and TEM (B) data supports the validity of this conclusion. This is also consistent with the previous observation at a higher monomer weight fraction (see section 1.5). The situation is much different for the miniemulsion containing TPO in the droplet core. In this case, droplets and particles exhibit similar size distributions whether determined by DLS ( $\left.\mathbf{A}^{\prime}\right)$ or TEM ( $\left.\mathbf{B}^{\prime}\right)$, and $N_{p} / N_{d}$ was estimated at 0.9 (DLS data). The fact that the number of particles derives directly from the number of droplets indicates a predominant droplet nucleation. The explanation for this effect is that water-insoluble initiator reduces the probability of forming oligoradicals in water, and therefore, the extent of homogeneous nucleation. The situation is quite different with TPO-Li, the water-soluble radicals are unlikely to be captured by the existing droplets/particles, leading to a predominant homogeneous nucleation.

\section{II.3 Effect of SDS concentration}

In this second series of experiments, the SDS concentration was varied $(3,6,12$ and $24 \mathrm{mM})$ as a means of obtaining a set of 4 types of stable droplets of different sizes. This has implied that in certain cases the surfactant concentration exceeds CMC ([SDS] $\geq 12 \mathrm{mM}$ ), therefore, micelles are present and micellar nucleation may occur. The resulting droplets have an intensityaverage diameter $\left(D_{d}\right)$ of $104-210 \mathrm{~nm}$. Based on DLS data, a systematic comparison of droplet and particle size distributions is presented in Table 1 and Figure 8. In the presence of the water-insoluble initiator TPO ( $\mathbf{A}^{\prime}, \mathbf{B}^{\prime}, \mathbf{C}^{\prime}$ and $\left.\mathbf{D}^{\prime}\right)$, a robust droplet nucleation operates regardless of the droplet size. With the water-soluble initiator TPO-Li (A, B, C and D), the
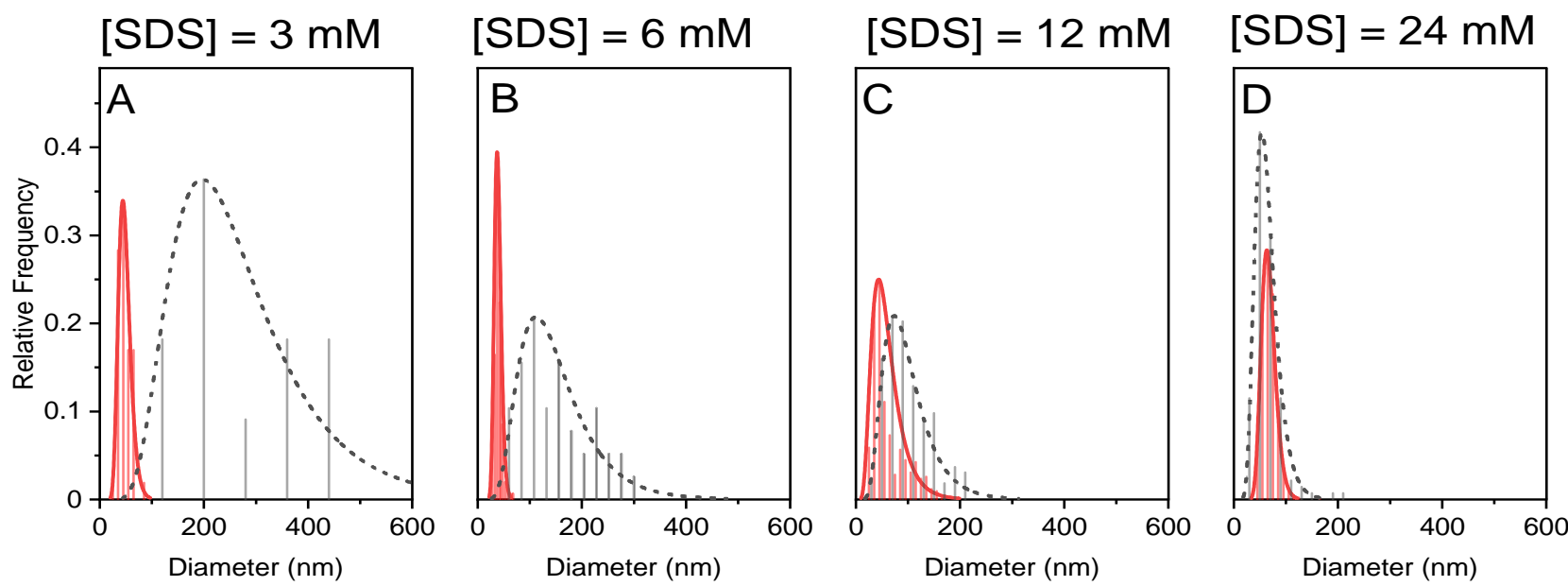

Figure 9. Effect of initiator solubility on particle size distribution (derived from NS-TEM imaging) of a latex derived from an EDDT-DAP miniemulsion (monomer content: 1 wt\%) prepared at different surfactant concentrations: Dotted line : TPO as an initiator ; solid line : TPO-Li as an initiator. 
extent of droplet nucleation increases appreciably as droplet size decreases. For the lowest droplet size $\left(D_{d}=104 \mathrm{~nm}\right)$, a good correspondence between particle and droplet size distribution and a $N_{p} / N_{d}$ ratio very close to 1 were found. It is likely that radical capture in particles can be enhanced by reducing the initial droplet size, improving droplet nucleation efficiency.

To evaluate the validity of this interpretation, particle size distributions were also evaluated from negative stained TEM images of the 8 latexes (Figure 9), four containing TPO and four other containing TPO-Li. A reasonable assumption based on the previous experimental results is to consider that the TPO-initiated systems undergo a prominent droplet nucleation, irrespective of surfactant concentration. Under these conditions, the particle size distribution should be the replica or the "one to one" copy" of the initial droplet size distribution. Based on this assumption, one can distinguish two behaviours for the TPO-Li-initiated systems in agreement with the DLS results. At low SDS concentration (A: $3 \mathrm{mM}$ and $\mathbf{B}: 6$ $\mathrm{mM}), \mathrm{a}$

Table 1. Influence of water-solubility of photoinitiators on nucleation mechanism Monomer content was kept at $1 \% \mathrm{wt} /$ aqueous phase, and PI content $2 \% \mathrm{wt} / \mathrm{monomer}$.

\begin{tabular}{|c|c|c|c|c|}
\hline \multirow{2}{*}{ Initiator } & $\begin{array}{c}{[\mathrm{CDS}]} \\
(\mathrm{mM})\end{array}$ & $D_{d}(\mathrm{~nm})$ & $D_{p}(\mathrm{~nm})$ & $N_{p} / N_{d}$ \\
\hline \multirow{3}{*}{ TPO-Li } & 24 & $104 \pm 2$ & $102 \pm 2$ & 1.1 \\
\cline { 2 - 5 } & 12 & $133 \pm 5$ & $103 \pm 5$ & 2.1 \\
\cline { 2 - 5 } & 6 & $183 \pm 6$ & $91 \pm 2$ & 8.2 \\
\cline { 2 - 5 } & 3 & $210 \pm 5$ & $154 \pm 5$ & 3.8 \\
\hline \multirow{3}{*}{ TPO } & 24 & $124 \pm 3$ & $127 \pm 2$ & 0.9 \\
\cline { 2 - 5 } & 12 & $138 \pm 1$ & $136 \pm 3$ & 1.0 \\
\cline { 2 - 5 } & 6 & $166 \pm 5$ & $173 \pm 5$ & 0.9 \\
\cline { 2 - 5 } & 3 & $221 \pm 5$ & $221 \pm 7$ & 1.0 \\
\hline
\end{tabular}

population of particle smaller than the starting droplet population (TPO-derived latex) suggests a substantial droplet nucleation. At high SDS concentration (C: $12 \mathrm{mM}$ and D: 24 $\mathrm{mM})$, the particle size distribution is somewhat similar to that of the droplets, suggesting a dominant droplet nucleation. The driving force is the increase probability of radical capture by monomer droplets when their diameter is reduced to about $100 \mathrm{~nm}$. Thus, when droplet size is small enough, the size distribution of particles is not dependent on whether the initiator is soluble in water.

\section{II.4 Effect of monomer solubility}

In miniemulsion radical chain polymerization, it is well established that the use of more water-soluble monomers can increase the occurrence of homogeneous nucleation by reducing radical desorption rate and nucleation duration. Thus, it is important to determine in what extent a similar dependency exists for miniemulsion thiol-ene polymerization. For this purpose, a range of thiol and ene monomers of different water solubility compared to the reference monomers EDDT and DAP have been employed (see chemical structures in Figure 1). For thiol monomers, the water insolubility decreases in the order: EDDT < thiodiethanethiol (DMDS) < hexadecanedithiol (HMDT). For the ene monomer, the order is following DAP < cyclohexyl divinylether (CHDM). The water-solubility values are given in the experimental section. By combining these monomers, four thiol-ene miniemulsions composed of a monomer phase of decreased water-insolubility have been prepared: EDDT-DAP (ref.) < DMDS-DAP < HMDT-DAP < HMDT-CHDM. The existence of stable and photolatent droplets was firstly ascertained. As shown in Figure 10, all TPO-initiated polymerizations show similar profiles of droplet and size distributions, suggesting that the droplet nucleation is not effected by the change of monomer water-solubility. More surprising is that homogeneous nucleation is operative in all systems initiated by TPO-Li, even when the most water-insoluble thiol-ene couple HMDT-CHDM is employed. A word of caution regarding this comparison is that the droplet size differs depending on the monomer composition, so direct comparison is not possible.
TPO-Li

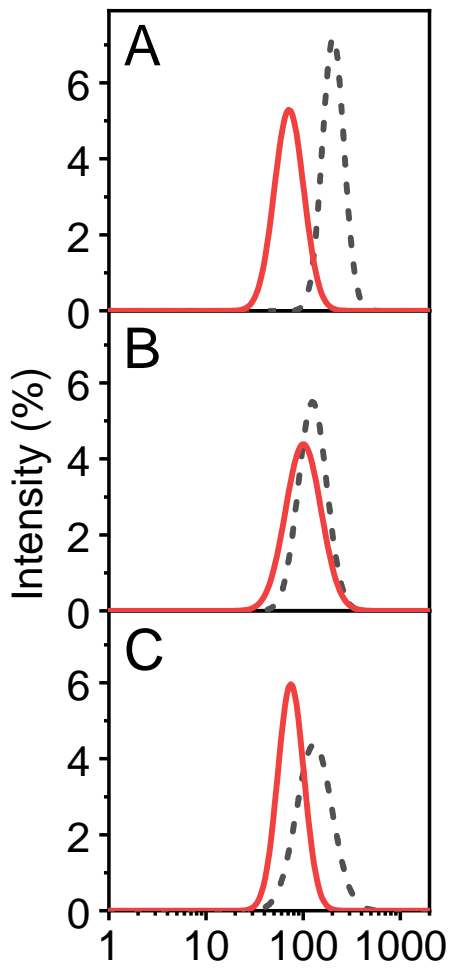

Diameter (nm)
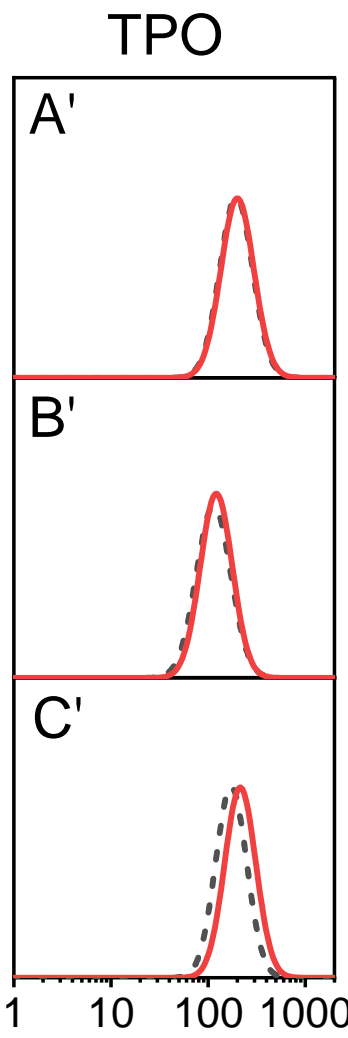

Diameter $(\mathrm{nm})$
Figure 10. DLS size distribution of latex and droplets derived from DAP-DMDS (A), DAPHMDT (B), and CHDM-HMDT (C) using TPO-Li as the water-soluble initiator and SDS as the surfactant $([S D S]=6 \mathrm{mM}) . A^{\prime}, B^{\prime}$ and $C^{\prime}$ are analogous distributions when the waterinsoluble initiator TPO was used.

\section{CONCLUSION}

A wide variety of thiol-ene monomers have been successfully polymerized by miniemulsion thiol-ene step photopolymerization in the literature, but the question of 
particle nucleation mechanism has remained elusive. This study lays the foundation for favouring droplet nucleation, which is the preferred nucleation mode in a miniemulsion polymerization. It has been postulated that the specific stepwise mechanism of a thiol-ene reaction could reduce the extent of droplet nucleation and increase the probability of forming polymer particles by homogeneous nucleation. These assumptions are justified on the basis that in a step polymerization the size of the polymer chains increases at a relatively slower pace compared to an analogous chain polymerization, resulting in extended stay of oligomeric radicals in the aqueous phase and higher radical desorption rate.

Involving a model EDDT-DAP diallyl ether-dithiol miniemulsion, the first part describes the great difficulty of obtaining a chemically stable miniemulsion. Thiol-ene monomer miniemulsions are highly susceptible to thermal selfinitiated polymerization in the dark because of facile production of adventitious radicals during sonication. The use of radical inhibitor at relatively high concentration (DBHQ at 50 $\mathrm{mM}$ in monomer) was found to be a practical solution for the EDTT-DAP system. However, a persistent problem is that concentration and type of radical inhibitor must be adjusted to each thiol-ene miniemulsion to impart long-term chemical stability. Efforts to overcome this problem will require a better understanding of the self-initiation mechanism to allow the prediction of thiol-ene monomer spontaneous reactivity. Another difficulty arises because of the poor monomer droplets stability. This was assigned to Ostwald ripening and to the limited solubility of HD (costabilizer) in the monomer mixture. The reader is also cautioned that a high thiol-ene droplet stability can be mistakenly achieved by the slow increase in molecular weight due to sonication-induced polymerization. In our case, the limited droplet stability of the EDDT-DAP miniemulsion did not prevent a subsequent polymerization. Reproducible kinetic data were achieved when the miniemulsion was photopolymerized in batch just after ultrasonication. Using a $20 \mathrm{~mL}$ sample with a $20 \mathrm{wt} \%$ monomer phase, high-molecular-weight products were obtained by employing a water-soluble initiator (TPO-Li) at $385 \mathrm{~nm}$. Despite broad droplet size distribution, large droplet size $(170 \mathrm{~nm})$ and moderate irradiance $\left(3.7 \mathrm{~mW} / \mathrm{cm}^{2}\right)$, the reaction times were reasonable (20 $\mathrm{min})$.

For an accurate determination of particle nucleation mode, thiol-ene droplets with a low monomer weight fraction (1 $w t \%)$ were prepared. Under these conditions, they possess chemical stability, droplet stability and monomodal droplet size distribution. Several reaction parameters must be controlled for droplet particle nucleation to proceed successfully. Using a water-soluble initiator, droplet nucleation generally coexists with homogeneous nucleation (mixed nucleation mode) except when the droplet size was reduced to $100 \mathrm{~nm}$. Conversely, a water-insoluble initiator results in a predominant droplet nucleation irrespective of droplet size and monomer water-solubility. Presumably, the driving forces of this robust droplet nucleation are the water-insolubility of the radicals (favouring a reaction confined to the droplets) and the high reaction rates (minimizing the mass transfer of monomer from droplets to particles). This suggests that using a water-insoluble initiator is the best way to promote a high droplet nucleation efficiency in miniemulsion thiol-ene photopolymerization. However, our approach is not without limitations. Firstly, the low used solids content can disfavour radical capture by droplets, and secondly, no quantitative analysis is proposed when a mixed nucleation mode is operative.

\section{GRAPHICAL ABSTRACT}

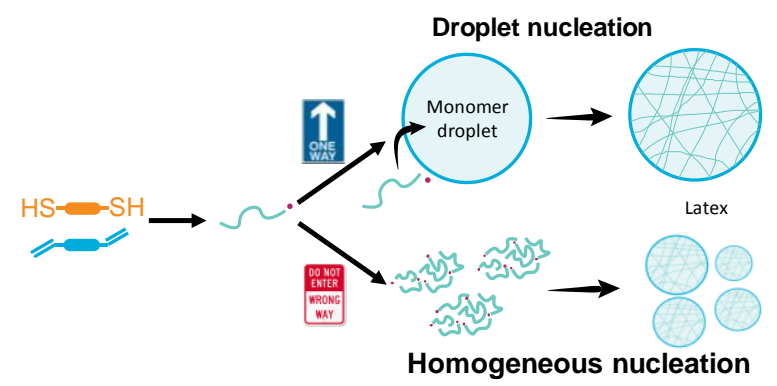

\section{CONFLICTS OF INTEREST}

There are no conflicts to declare.

\section{ACKNOWLEDGEMENTS}

This project has received funding from the European Union's Horizon 2020 research and innovation programme under the Marie Skłodowska-Curie grant agreement No 765341 (Project PHOTO-EMULSION, MSCA-ITN-2017).

\section{NOTES AND REFERENCES}

1 F. Denes, M. Pichowicz, G. Povie and P. Renaud, Chem. Rev., 2014, 114, 2587-2693.

2 B. Cramer and C. N. Bowman, in Chemoselective and Bioorthogonal Ligation Reactions, eds. W. R. Algar, P. E. Dawson and I. L. Medintz, Wiley-VCH Verlag, Weinheim, Germany, 2017, pp. 117-145.

3 J. Lalevée and J.-P. Fouassier, Eds., Photopolymerisation Initiating Systems, RSC, Cambridge, UK, 2018.

4 R. Schwalm, in UV Coatings, Elsevier, Amsterdam, Netherlands, 2007, pp. 19-61.

5 N. B. Cramer and C. N. Bowman, in Polymer Chemistry Series, eds. A. Lowe and C. Bowman, Royal Society of Chemistry, Cambridge, UK, 2013, pp. 1-27.

6 T. O. Machado, C. Sayer and P. H. H. Araujo, Eur. Polym. J, 2017, 86, 200-215.

7 Y. Zhang, S. Liu, T. Li, L. Zhang, U. Azhar, J. Ma, C. Zhai, C. Zong and S. Zhang, Carbohydr. Polym., 2020, 236, 116021.

8 W. Fu, L. Wang and J. He, J. Mech. Behav. Biomed. Mater., 2019, 95, 53-59.

9 A. M. van Herk and R. G. Gilbert, in Chemistry and Technology of Emulsion Polymerisation, ed. A. M. van Herk, John Wiley \& Sons Ltd, Oxford, UK, 2013, pp. 43-73. 
10 A. Javadi, A. Cobaj and M. D. Soucek, in Handbook of Waterborne Coatings, Elsevier, Amsterdam, Netherlands, 2020, pp. 303-344.

11 F. Deubel, V. Bretzler, R. Holzner, T. Helbich, O. Nuyken, B. Rieger and R. Jordan, Macromol. Rapid Commun., 2013, 34, 1020-1025.

12 C. M. Quoc Le, M. Schmutz and A. Chemtob, Macromolecules, 2020, 53, 2369-2379.

13 F. Jasinski, P. B. Zetterlund, A. M. Braun and A. Chemtob, Prog. Polym. Sci., 2018, 84, 47-88.

14 O. Z. Durham and D. A. Shipp, Polym. Rev., 2020, 0, 1-26.

15 F. Jasinski, E. Lobry, B. Tarablsi, A. Chemtob, C. CroutxeBarghorn, D. Le Nouen and A. Criqui, ACS Macro Lett., 2014, 3 958-962.

16 F. Jasinski, A. Rannee, J. Schweitzer, D. Fischer, E. Lobry, C Croutxe-Barghorn, M. Schmutz, D. Le Nouen, A. Criqui and A. Chemtob, Macromolecules, 2016, 49, 1143-1153.

17 D. V. Amato, D. N. Amato, A. S. Flynt and D. L. Patton, Polym. Chem., 2015, 6, 5625-5632.

18 M. Taghavikish, S. Subianto, N. K. Dutta, L. de Campo, J. P. Mata, C. Rehm and N. R. Choudhury, ACS omega, 2016, 1, 2940.

19 A. C. de Meneses, T. O. Machado, C. Sayer, P. H. H. Araújo and D. de Oliveira, Braz. J. Chem. Eng., 2018, 35, 691-698.

20 A. C. de Meneses, P. C. M. dos Santos, T. O. Machado, C. Sayer, D. de Oliveira and P. H. Hermes de Araújo, J. Polym. Res. 2017, 24, 202.

21 T. O. Machado, P. B. Cardoso, P. E. Feuser, C. Sayer and P. H. Araújo, Colloids Surf., $B, 2017,159$, 509-517.

22 P. B. Cardoso, T. O. Machado, P. E. Feuser, C. Sayer, M. A. Meier and P. H. Araújo, Eur. J. Lipid Sci. Technol., 2018, 120 1700212 .

23 F. Hoelscher, T. O. Machado, D. de Oliveira, P. H. Hermes de Araújo and C. Sayer, Polym. Degradation Stability, 2018, 156 211-217.

24 P. C. M. Santos, T. O. Machado, J. V. C. Santin, P. E. Feuser, E. S. Córneo, R. A. Machado-de-Ávila, C. Sayer and P. H. H. Araújo J. Appl. Polym. Sci., 2021, 138, 49741

25 K. Jiang, Y. Liu, Y. Yan, S. Wang, L. Liu and W. Yang, Polym. Chem., 2017, 8, 1404-1416.

26 F. Alimohammadi, C. Wang, O. Z. Durham, H. R. Norton, C. N. Bowman and D. A. Shipp, Polymer, 2016, 105, 180-186.

27 O. Z. Durham, D. V. Chapman, S. Krishnan and D. A. Shipp, Macromolecules, 2017, 50, 775-783.

28 D. Liu, B. Yu, X. Jiang and J. Yin, Langmuir, 2013, 29, 53075314.

29 O. Z. Durham and D. A. Shipp, Colloid Polym. Sci., 2015, 293, 2385-2394.

30 O. Z. Durham, H. R. Norton and D. A. Shipp, RSC Adv., 2015, 5, 66757-66766.

31 K. J. Cassidy, O. Z. Durham and D. A. Shipp, Macromol. React. Eng., 2019, 13, 1800075.

32 P. Muhsir, E. Çakmakçi, S. Demir and A. Ogan, Colloids Surf., $B, 2018,170,122-128$.

33 J. M. Asua, in Polymer Reaction Engineering of Dispersed Systems: Volume II, ed. W. Pauer, Springer International Publishing, Cham, Switzerland, 2018, pp. 1-22.

34 A. B. Lowe, Polym. Chem., 2014, 5, 4820-4870.

35 P. J. Scott, C. R. Kasprzak, K. D. Feller, V. Meenakshisundaram, C. B. Williams and T. E. Long, Polym. Chem., 2020, 11, 3498-3524.

36 F. J. Schork, Y. Luo, W. Smulders, J. P. Russum, A. Butté and K. Fontenot, Miniemulsion Polymerization, in Polymer Particles, ed. M. Okubo, Advances in Polymer Science, vol. 175. Springer, Berlin, Heidelberg.

37 C. S. Chern, Y. C. Liou and T. J. Chen, Macromol. Chem. Phys. 1998, 199, 1315-1322.
38 Y. Luo and F. J. Schork, J. Polym. Sci., Part A: Polym. Chem., 2002, 40, 3200-3211.

39 R. Rodríguez, M. J. Barandiaran and J. M. Asua, Macromolecules, 2007, 40, 5735-5742.

40 G. Odian, in Principles of Polymerization, Fourth Ed., John Wiley \& Sons, Ltd, Hoboken, USA, 2004, pp. 39-197.

41 N. Nauman, N. Zaquen, T. Junkers, C. Boyer and P. B. Zetterlund, Macromolecules, 2019, 52, 4492-4499.

42 J. M. Asua, Prog. Polym. Sci., 2002, 27, 1283-1346.

43 E. Klemm, S. Sensfuß, U. Holfter and H. J. Flammersheim, Angew. Makromol. Chem., 1993, 212, 121-127.

44 N. B. Cramer, J. P. Scott and C. N. Bowman, Macromolecules, 2002, 35, 5361-5365.

45 S. Sensfuß, M. Friedrich and E. Klemm, Makromol. Chem., 1991, 192, 2895-2900.

46 H. H. Szmant, A. J. Mata, A. J. Namis and A. M. Panthananickal, Tetrahedron, 1976, 32, 2665-2680.

47 M. Manea, A. Chemtob, M. Paulis, J. C. de la Cal, M. J. Barandiaran and J. M. Asua, AlChE J., 2008, 54, 289-297.

48 B. A. Bhanvase, D. V. Pinjari, S. H. Sonawane, P. R. Gogate and A. B. Pandit, Ultrason. Sonochem., 2012, 19, 97-103.

49 B. M. Teo, S. W. Prescott, M. Ashokkumar and F. Grieser, Ultrason. Sonochem., 2008, 15, 89-94.

50 P. Esfandiari, S. C. Ligon, J. J. Lagref, R. Frantz, Z. Cherkaou and R. Liska, J. Polym. Sci. Polym. Chem., 2013, 51, 4261-4266.

51 C. M. Miller, P. J. Blythe, E. D. Sudol, C. A. Silebi and M. S. ElAasser, J. Polym. Sci. Polym. Chem., 1994, 32, 2365-2376.

52 K. Landfester, N. Bechthold, F. Tiarks and M. Antonietti, Macromolecules, 1999, 32, 5222-5228.

53 E. Poggi and J.-F. Gohy, Colloid Polym. Sci., 2017, 295, 20832108.

54 S.-H. Hu and X. Gao, J. Am. Chem. Soc., 2010, 132, 72347237.

55 N. B. Cramer, S. K. Reddy, A. K. O'Brien and C. N. Bowman, Macromolecules, 2003, 36, 7964-7969.

56 P. Renz, M. Kokkinopoulou, K. Landfester and I. Lieberwirth, Macromol. Chem. Phys., 2016, 217, 1879-1885.

57 L. E. Franken, E. J. Boekema and M. C. A. Stuart, Adv. Sci., 2017, 4, 1600476.

58 C. A. Scarff, M. J. G. Fuller, R. F. Thompson and M. G. Iadaza, J. Visualized Exp., DOI:10.3791/57199. 